\title{
SEMICLASSICAL ASYMPTOTICS \\ AND GAPS IN THE SPECTRA \\ OF PERIODIC SCHRÖDINGER OPERATORS WITH MAGNETIC WELLS
}

\author{
BERNARD HELFFER AND YURI A. KORDYUKOV
}

\begin{abstract}
We show that, under some very weak assumption of effective variation for the magnetic field, a periodic Schrödinger operator with magnetic wells on a noncompact Riemannian manifold $M$ such that $H^{1}(M, \mathbb{R})=0$, equipped with a properly disconnected, cocompact action of a finitely generated, discrete group of isometries, has an arbitrarily large number of spectral gaps in the semi-classical limit.
\end{abstract}

\section{INTRODUCTION}

Let $M$ be a noncompact oriented manifold of dimension $n \geq 2$ equipped with a properly disconnected action of a finitely generated, discrete group $\Gamma$ such that $M / \Gamma$ is compact. Suppose that $H^{1}(M, \mathbb{R})=0$, i.e. any closed 1 -form on $M$ is exact. Let $g$ be a $\Gamma$-invariant Riemannian metric and $\mathbf{B}$ a real-valued $\Gamma$-invariant closed 2-form on $M$. Assume that $\mathbf{B}$ is exact and choose a real-valued 1-form $\mathbf{A}$ on $M$ such that $d \mathbf{A}=\mathbf{B}$.

Consider a Schrödinger operator with magnetic potential A,

$$
H^{h}=(i h d+\mathbf{A})^{*}(i h d+\mathbf{A}),
$$

as a self-adjoint operator in the Hilbert space $L^{2}(M)$. Here $h>0$ is a semiclassical parameter, which is assumed to be small.

For any $x \in M$ denote by $B(x)$ the anti-symmetric linear operator on the tangent space $T_{x} M$ associated with the 2 -form $\mathbf{B}$ :

$$
g_{x}(B(x) u, v)=\mathbf{B}_{x}(u, v), \quad u, v \in T_{x} M .
$$

Recall that the intensity of the magnetic field is defined as

$$
\operatorname{Tr}^{+}(B(x))=\sum_{\substack{\lambda_{j}(x)>0 \\ i \lambda_{j}(x) \in \sigma(B(x))}} \lambda_{j}(x)=\frac{1}{2} \operatorname{Tr}\left(\left[B^{*}(x) \cdot B(x)\right]^{1 / 2}\right) .
$$

Received by the editors December 21, 2005 and, in revised form, September 12, 2006.

2000 Mathematics Subject Classification. Primary 35P20, 35J10, 47F05, 81Q10.

The first author acknowledges support from the SPECT programme of the ESF and from the European Research Network 'Postdoctoral Training Program in Mathematical Analysis of Large Quantum Systems' with contract number HPRN-CT-2002-00277.

The second author acknowledges support from the Russian Foundation of Basic Research (grant no. 04-01-00190). 
Let

$$
b_{0}=\min \left\{\operatorname{Tr}^{+}(B(x)): x \in M\right\} .
$$

We will always assume that there exist a (connected) fundamental domain $\mathcal{F}$ and $\epsilon_{0}>0$ such that

$$
\operatorname{Tr}^{+}(B(x)) \geq b_{0}+\epsilon_{0}, \quad x \in \partial \mathcal{F}
$$

For any $\epsilon_{1} \leq \epsilon_{0}$, let

$$
U_{\epsilon_{1}}=\left\{x \in \mathcal{F}: \operatorname{Tr}^{+}(B(x))<b_{0}+\epsilon_{1}\right\} .
$$

Thus $U_{\epsilon_{1}}$ is an open subset of $\mathcal{F}$ such that $U_{\epsilon_{1}} \cap \partial \mathcal{F}=\emptyset$ and, for $\epsilon_{1}<\epsilon_{0}, \overline{U_{\epsilon_{1}}}$ is compact and included in the interior of $\mathcal{F}$. Any connected component of $U_{\epsilon_{1}}$ with $\epsilon_{1}<\epsilon_{0}$ can be understood as a magnetic well (attached to the effective potential $\left.h \cdot \operatorname{Tr}^{+}(B(x))\right)$.

Consider the set $U_{\epsilon_{0}}^{+}$, which consists of all $x \in U_{\epsilon_{0}}$ such that the rank of $B(x)$ is locally constant at $x$, that is, constant in an open neighborhood of $x$. Let us assume that

$$
\operatorname{Tr}^{+} B \text { is not locally constant on } U_{\epsilon_{0}}^{+} \text {. }
$$

The assumption (1.2) holds for any $B$, satisfying the assumption (1.1), if the dimension $n$ equals 2 or 3 .

If $T$ is a self-adjoint operator, $\sigma(T)$ denotes its spectrum. By a gap in the spectrum of $T$ we will mean a maximal interval $(a, b)$ such that

$$
(a, b) \cap \sigma(T)=\emptyset \text {. }
$$

Theorem 1.1. Under the assumptions (1.1) and (1.2), there exists, for any natural $N, h_{0}>0$ such that, for any $h \in\left(0, h_{0}\right]$, the spectrum of $H^{h}$ contained in $\left[0, h\left(b_{0}+\epsilon_{0}\right)\right]$ has at least $N$ gaps.

The proof of Theorem 1.1 is based on the study of the tunneling effect for the operator $H^{h}$. First, we prove that the spectrum of $H^{h}$ is localized inside an exponentially small neighborhood of the spectrum of its Dirichlet realization $H_{D}^{h}$ in $D=\overline{U_{\varepsilon_{1}}}$ for $\varepsilon_{1}<\varepsilon_{0}$ (a multi-well problem). For this, we follow the approach to the study of the tunneling effect in multi-well problems developed by Helffer and Sjöstrand for Schrödinger operators with electric potentials (see for instance $[8,9]$ ) and extended to magnetic Schrödinger operators in $[10,7]$. Since $H^{h}$ is not with compact resolvent, we work not with individual eigenfunctions as in [8], but with resolvents, using the strategy developed in $[9,11,5,3]$ for the case of an electric potential and in [6] for the case of a magnetic field. The idea is to construct an approximate resolvent $R^{h}(z)$ of the operator $H^{h}$ for any $z$, which is not exponentially close to the spectrum of $H_{D}^{h}$, starting from the resolvent of $H_{D}^{h}$ and the resolvent of the Dirichlet realization of $H^{h}$ in the complement to the wells. The proof of the fact that the error of the approximation is exponentially small is based on Agmon-type weighted estimates (cf. [1] and their semi-classical versions in [8] for the case of Schrödinger operators and [7] for the case of magnetic Schrödinger operators). A very related result was proved by Nakamura in [18].

Thus the proof is reduced to the study of the discrete spectrum of the operator $H_{D}^{h}$ in the interval $\left[0, h\left(b_{0}+\epsilon_{0}\right)\right]$. Actually, it remains to show that there is, as $h \rightarrow 0$, an arbitrarily large number of gaps in the spectrum of $H_{D}^{h}$ of size $>h^{M}$ with some constant $M>0$. For this, we use a weak polynomial upper bound on the number of eigenvalues of $H_{D}^{h}$ contained in $\left[0, h\left(b_{0}+\epsilon_{0}\right)\right]$ and the construction of 
quasimodes of the operator $H^{h}$ given in the proof of [7, Theorem 2.2]. We need the fact that the number of quasimodes, which we can construct, is sufficiently large, and that follows from a slight modification of [7, Theorem 2.2] (see Proposition 2.3 below) and makes an essential use of the assumption (1.2).

It seems that the periodicity assumption in Theorem 1.1 is not important, and the theorem could probably be extended to the case when we only assume the existence of an infinite number of identical magnetic wells of the form $U_{\epsilon_{0}}$ separated by regions where the estimate (1.1) holds. To show such a result, one should use the strategy developed in [3] in the case of the strong electric field and in [4] for the tight binding model, but this will not be detailed in this article. $H^{h}$.

Let us mention some previous results on gaps in the spectrum of the operator

An asymptotic description of the spectrum of the two-dimensional magnetic Schrödinger operator with a periodic potential in a strong magnetic field can be given, using averaging methods or effective Hamiltonians together with semiclassical analysis (see, for instance, $[2,11,12]$ and the references therein). This allows us to give, at least heuristically, a more precise asymptotic picture of spectral bands and gaps for these operators. However, it should be noted that, in these papers, the magnetic field is, usually, supposed to be uniform and spectral gaps are created by the electric potential, whereas in our case the electric field vanishes and spectral gaps are created by a periodic array of magnetic barriers.

In [13], Hempel and Herbst studied the strong magnetic field limit $(\lambda \rightarrow \infty)$ for the periodic Schrödinger operator in $\mathbb{R}^{n}$ :

$$
H_{\lambda \mathbf{A}, 0}=(D-\lambda \mathbf{A})^{2}, \quad D_{j}=\frac{1}{i} \frac{\partial}{\partial x_{j}},
$$

where $\mathbf{B}=d \mathbf{A}$ is a $\mathbb{Z}^{n}$-periodic 2-form. Let $S=\left\{x \in \mathbb{R}^{n}: \mathbf{B}(x)=0\right\}$ and $S_{\mathbf{A}}=\left\{x \in \mathbb{R}^{n}: \mathbf{A}(x)=0\right\}$. Assume that the set $S \backslash S_{\mathbf{A}}$ has measure zero, the interior of $S$ is non-empty and $S$ can be represented as $S=\bigcup_{j \in \mathbb{Z}^{n}} S_{j}$ (up to a set of measure zero) where the $S_{j}$ are pairwise disjoint compact sets with $S_{j}=S_{0}+j$. It is shown that, as $\lambda \rightarrow \infty, H_{\lambda \mathbf{A}, 0}$ converges in the norm resolvent sense to the Dirichlet Laplacian $-\Delta_{S}$ on the closed set $S$. Therefore, as $\lambda \rightarrow \infty$, the spectrum of $H_{\lambda \mathbf{A}, 0}$ concentrates around the eigenvalues of $-\Delta_{S}$ and gaps open up in the spectrum of $H_{\lambda \mathbf{A}, 0}$. For the operator $H^{h}=h^{2} H_{h^{-1}} \mathbf{A}, 0$ this means that for any natural $N$ there exist $C>0$ and $h_{0}>0$ such that the part of the spectrum of $H^{h}$ contained in the interval $\left[0, C h^{2}\right]$ has at least $N$ spectral gaps for any $h \in\left(0, h_{0}\right)$. The rate of approach of the resolvent $\left(H_{\lambda \mathbf{A}, 0}+1\right)^{-1}$ to a limit was studied by Nakamura in $[18]$.

The case when the set $S \backslash S_{\mathrm{A}}$ has nonzero measure was studied by Herbst and Nakamura in [14]. They showed that in many situations of interest where this condition holds the equivalence class of $H_{\lambda \mathbf{A}, 0}$ approaches a periodic or almostperiodic orbit in the space of such classes as $\lambda \rightarrow \infty$, and, therefore, the spectrum of $H_{\lambda \mathbf{A}, 0}$ approaches a periodic or almost-periodic orbit in the space of subsets of $[0, \infty)$.

In [16], the author investigated the case when the bottom $S$ of magnetic wells has measure zero and the magnetic field has regular behavior near $S$. More precisely, assume that there exists at least one zero of $B$, and, for some integer $k>0$, if $B\left(x_{0}\right)=0$, then there exists a positive constant $C$ such that, for all $x$ in some 
neighborhood of $x_{0}$,

$$
C^{-1}\left|x-x_{0}\right|^{k} \leq \operatorname{Tr}^{+}(B(x)) \leq C\left|x-x_{0}\right|^{k} .
$$

It is shown in [16] that, under these assumptions, there exists an increasing sequence $\left\{\lambda_{m}, m \in \mathbb{N}\right\}$, satisfying $\lambda_{m} \rightarrow \infty$ as $m \rightarrow \infty$, such that, for any $a$ and $b$, satisfying $\lambda_{m}<a<b<\lambda_{m+1}$ with some $m$,

$$
\left[a h^{\frac{2 k+2}{k+2}}, b h^{\frac{2 k+2}{k+2}}\right] \cap \sigma\left(H^{h}\right)=\emptyset,
$$

for any $h>0$ small enough. In particular, this implies that, for any natural number $N$, there exist $C>0$ and $h_{0}>0$ such that the part of the spectrum of $H^{h}$ contained in the interval $\left[0, C h^{\frac{2 k+2}{k+2}}\right]$ has at least $N$ gaps for any $h \in\left(0, h_{0}\right)$.

The results of this paper can be considered as a complement of the results of [16] and, in some sense, correspond to the case when the condition (1.3) holds with $k=0$ (whereas the results of [13] are related with the case when the condition (1.3) holds with arbitrarily large $k$ ). From the other side, it should be noted that here we state only the existence of an arbitrarily large number of spectral gaps in the semi-classical limit and don't know of any results on the spectral concentration in this case.

The authors are grateful to the referee for useful remarks.

\section{Proof of the Main theorem}

For any domain $W$ in $M$, denote by $H_{W}^{h}$ the operator $H^{h}$ in $\bar{W}$ with the Dirichlet boundary conditions. The operator $H_{W}^{h}$ is generated by the quadratic form

$$
q_{W}^{h}[u]:=\int_{W}|(i h d+\mathbf{A}) u|^{2} d x
$$

with the domain

$$
\operatorname{Dom}\left(q_{W}^{h}\right)=\left\{u \in L^{2}(W):(i h d+\mathbf{A}) u \in L^{2} \Omega^{1}(W),\left.u\right|_{\partial W}=0\right\},
$$

where $L^{2} \Omega^{1}(W)$ denotes the Hilbert $L^{2}$-space of differential 1-forms on $W$, and $d x$ is the Riemannian volume form on $M$.

Let us assume that (1.1) and (1.2) are satisfied. By (1.2), there exists a connected open set $\Omega \subset U_{\epsilon_{0}}$ such that the rank of $B(x)$ is constant on $\Omega$ and $\operatorname{Tr}^{+} B(\bar{\Omega})=$ $[\alpha, \beta], \alpha<\beta$. Without loss of generality, we can assume that $\Omega \subset U_{\epsilon_{1}}$ for some $\epsilon_{1}<\epsilon_{0}$ and, therefore, $[\alpha, \beta] \subset\left[0, b_{0}+\epsilon_{1}\right]$.

For a fixed $\epsilon_{2}$ such that $\epsilon_{1}<\epsilon_{2}<\epsilon_{0}$, consider the operator $H_{D}^{h}$ associated with $D=\overline{U_{\epsilon_{2}}}$. The operator $H_{D}^{h}$ has a discrete spectrum. Denote by $\lambda_{1}^{h}<\lambda_{2}^{h}<\ldots<$ $\lambda_{N(h)}^{h}$ the eigenvalues of $H_{D}^{h}$ contained in the interval $[h \alpha, h \beta]$. It follows from rough estimates for the eigenvalue counting function of $H_{D}^{h}$ (cf. for instance [7, Lemma 4.2]) that there exist $C$ and $h_{0}$ such that

$$
N(h) \leq C h^{-n}, \quad \forall h \in\left(0, h_{0}\right] .
$$

Theorem 2.1. Under the assumption (1.1), for any $\epsilon_{1}<\epsilon_{2}<\epsilon_{0}$, there exist $C, c, h_{0}>0$ such that for any $h \in\left(0, h_{0}\right]$,

$$
\sigma\left(H^{h}\right) \cap\left[0, h\left(b_{0}+\epsilon_{1}\right)\right] \subset\left\{\lambda \in\left[0, h\left(b_{0}+\epsilon_{1}\right)\right]: \operatorname{dist}\left(\lambda, \sigma\left(H_{D}^{h}\right)\right)<C e^{-c / \sqrt{h}}\right\} .
$$


The proof of Theorem 2.1 will be given in Section 3. A slightly weaker version of this theorem (which involves the largest absolute value of the eigenvalues of $B(x)$ instead of $\left.\operatorname{Tr}^{+}(B(x))\right)$ was proved in [18].

By Theorem 2.1, $\sigma\left(H^{h}\right) \cap[h \alpha, h \beta]$ is contained in exponentially small neighborhoods of $\lambda_{j}^{h}, j=1,2, \cdots, N(h)$ : there exist $C, c, h_{0}>0$ such that for any $h \in\left(0, h_{0}\right]$,

$$
\sigma\left(H^{h}\right) \cap[h \alpha, h \beta] \subset \bigcup_{j=1}^{N(h)}\left[\lambda_{j}^{h}-C e^{-c / \sqrt{h}}, \lambda_{j}^{h}+C e^{-c / \sqrt{h}}\right] .
$$

It follows from (2.2) that for any $j$ such that $\lambda_{j+1}^{h}-\lambda_{j}^{h} \geq h^{M}$ with some $M>0$ the interval $\left(\lambda_{j}^{h}+C e^{-c / \sqrt{h}}, \lambda_{j+1}^{h}-C e^{-c / \sqrt{h}}\right)$ is a gap in the spectrum of $H^{h}$ if $h$ is small enough. Therefore, the proof of Theorem 1.1 is completed by the following fact.

Proposition 2.2. There exists a constant $M>0$ such that the number of $j \in$ $\{1,2, \cdots, N(h)-1\}$ with $\lambda_{j+1}^{h}-\lambda_{j}^{h} \geq h^{M}$ tends to infinity as $h \rightarrow 0$.

Proof. First, observe that there exists a constant $C_{1}>0$ such that, for any $j=$ $1,2, \cdots, N(h)-1$, we have

$$
\lambda_{j+1}^{h}-\lambda_{j}^{h} \leq C_{1} h^{4 / 3}
$$

and also

$$
\lambda_{1}^{h}-h \alpha \leq C_{1} h^{4 / 3}, \quad h \beta-\lambda_{N(h)}^{h} \leq C_{1} h^{4 / 3} .
$$

To see this, we will use the following proposition, which is a slight modification of [7, Theorem 2.2].

Proposition 2.3. Assume that the rank of $B$ is constant in a connected open subset $\Omega$. For any compact subset $K$ of $\Omega$, there exists $C>0$ such that, for any $\mu$ in $\operatorname{Tr}^{+} B(K)$ and for any $h \in(0,1]$,

$$
\left(-h^{4 / 3} C+h \mu, h \mu+h^{4 / 3} C\right) \cap \sigma\left(H_{D}^{h}\right) \neq \emptyset .
$$

Proof. We will follow the proof of [7, Theorem 2.2]. Denote by $2 d$ the rank of $B(y)$, $y \in \Omega$. By assumption, $d$ is independent of $y$. For any $y \in \Omega$, there exists an orthonormal base $e_{1}(y), e_{2}(y), \cdots, e_{n}(y)$ in $T_{y} M$ such that

$$
\begin{aligned}
B(y) e_{2 j-1}(y)=\mu_{j}(y) e_{2 j}(y), & j=1,2, \cdots, d, \\
B(y) e_{2 j}(y)=-\mu_{j}(y) e_{2 j-1}(y), & j=1,2, \cdots, d, \\
B(y) e_{2 d+k}(y)=0, & k=1,2, \cdots, n-2 d .
\end{aligned}
$$

Moreover, for any $j, \mu_{j}(y)$ depends continuously on $y \in \Omega$, and one can choose the orthonormal base $e_{1}(y), e_{2}(y), \cdots, e_{n}(y)$, depending continuously on $y$. Let $\phi_{y}: \mathcal{V}_{y} \rightarrow \mathbb{R}^{n}$ be a local coordinate chart given by the normal coordinates of the metric $g$ associated with the orthonormal base $e_{1}(y), e_{2}(y), \cdots, e_{n}(y)$. Without loss of generality, we can assume that $\phi_{y}$ is defined in a neighborhood $\mathcal{V}_{y} \subset \Omega$ of $y$ and $\phi_{y}\left(\mathcal{V}_{y}\right)$ is a fixed ball $B$ in $\mathbb{R}^{n}$ centered at the origin. Moreover, the family $\left\{\phi_{y}^{-1}: y \in \Omega\right\}$ yields a continuous family of smooth maps from $B$ to $M$. In the coordinates $\phi_{y}, g_{y}$ becomes the standard Euclidean metric on $\mathbb{R}^{n}$ and

$$
\mathbf{B}(y)=\sum_{j=1}^{d} \mu_{j}(y) d x_{2 j-1} \wedge d x_{2 j} .
$$


Now one can proceed as in the proof of [7, Theorem 2.2] and construct a continuous family $u_{y}^{h} \in C_{c}^{\infty}\left(\mathcal{V}_{y}\right) \subset L^{2}(D), y \in \Omega$, such that

$$
\left\|\left(H^{h}-h \operatorname{Tr}^{+}(B(y))\right) u_{y}^{h}\right\|_{L^{2}(D)} \leq C h^{4 / 3}\left\|u_{y}^{h}\right\|_{L^{2}(D)}, \quad y \in K,
$$

where $C$ is independent of $y \in K$ by continuity in $y$, that immediately concludes the proof.

By Proposition 2.3, the operator $H_{D}^{h}$ cannot have spectral gaps of size greater than $C_{1} h^{4 / 3}$ with some $C_{1}>0$, in the interval $[h \alpha, h \beta]$, that immediately implies the estimates (2.3) and (2.4).

Now assume from the contrary that for any real $M$ the cardinality of the set

$$
\mathcal{J}_{M}^{h}=\left\{j \in\{1,2, \cdots, N(h)-1\}: \lambda_{j+1}^{h}-\lambda_{j}^{h} \geq h^{M}\right\}
$$

is bounded as $h \rightarrow 0$ :

$$
\sharp \mathcal{J}_{M}^{h} \leq K, \quad h \in(0,1],
$$

where $K$ is independent of $h$. Then, using (2.1), (2.3), (2.4) and (2.5), we get, for all sufficiently small $h>0$,

$$
\begin{gathered}
h(\beta-\alpha)=\left(h \beta-\lambda_{N}^{h}\right)+\sum_{j=1}^{N-1}\left(\lambda_{j+1}^{h}-\lambda_{j}^{h}\right)+\left(\lambda_{1}^{h}-h \alpha\right) \\
=\left(h \beta-\lambda_{N}^{h}\right)+\sum_{j \in \mathcal{J}_{M}^{h}}\left(\lambda_{j+1}^{h}-\lambda_{j}^{h}\right)+\sum_{j \notin \mathcal{J}_{M}^{h}}\left(\lambda_{j+1}^{h}-\lambda_{j}^{h}\right)+\left(\lambda_{1}^{h}-h \alpha\right) \\
\leq C_{1} h^{4 / 3}+K C_{1} h^{4 / 3}+C h^{-n} h^{M}+C_{1} h^{4 / 3} .
\end{gathered}
$$

Taking $M>n+1$, we come to a contradiction.

\section{ExPONENTIAL LOCALIZATION OF THE SPECTRUM}

This section is devoted to the proof of Theorem 2.1. Throughout this section, we will assume that (1.1) is satisfied.

3.1. Weighted $L^{2}$-spaces. Let $W$ be an open domain (with regular boundary) in $M$. Let

$$
b_{0}(W)=\min \left\{\operatorname{Tr}^{+}(B(x)): x \in W\right\} .
$$

Denote by $C^{0,1}(\bar{W}, \mathbb{R})$ the class of uniformly Lipschitz continuous, real-valued functions on $\bar{W}$. Introduce the following class of weights:

$$
\mathcal{W}(\bar{W})=\left\{\Phi \in C^{0,1}(\bar{W}, \mathbb{R}): \underset{x \in \bar{W}}{\operatorname{ess}-\inf }\left(\operatorname{Tr}^{+} B(x)-b_{0}(W)-|\nabla \Phi(x)|^{2}\right)>0\right\} .
$$

Examples of functions in the class $\mathcal{W}(\bar{W})$ are given by the functions $f(x)=$ $(1-\epsilon) d_{W}(x, X)$, with an arbitrary $0<\epsilon \leq 1$ and $X \subset W$, where $d_{W}(x, y)$ is the distance associated with the (degenerate) Agmon metric

$$
\left[\operatorname{Tr}^{+}(B(x))-b_{0}(W)\right]_{+} \cdot g
$$

and, for any $x \in \mathbb{R}, x_{+}=\max (x, 0)$.

For any $\Phi \in \mathcal{W}(\bar{W})$ and $h>0$ define the Hilbert space

$$
L_{\Phi / \sqrt{h}}^{2}(W)=\left\{u \in L_{l o c}^{2}(W): e^{\Phi / \sqrt{h}} u \in L^{2}(W)\right\}
$$

with the norm

$$
\|u\|_{\Phi / \sqrt{h}}=\left\|e^{\Phi / \sqrt{h}} u\right\|, \quad u \in L_{\Phi / \sqrt{h}}^{2}(W),
$$


where $\|\cdot\|$ denotes the norm in $L^{2}(W)$ :

$$
\|u\|=\left(\int_{W}|u(x)|^{2} d x\right)^{1 / 2}, \quad u \in L^{2}(W) .
$$

By $\|\cdot\|_{\Phi / \sqrt{h}}$ we will also denote the norm of a bounded operator in $L_{\Phi / \sqrt{h}}^{2}(W)$.

Recall the following important identity (cf. for instance [7]).

Lemma 3.1. Let $W \subset M$ be an open domain (with $C^{2}$ boundary) and $\Phi \in$ $C^{0,1}(\bar{W}, \mathbb{R})$. For any $h>0, z \in \mathbb{C}$ and $u \in \operatorname{Dom}\left(H_{W}^{h}\right)$ one has

$$
\begin{aligned}
\operatorname{Re} \int_{W} e^{2 \Phi / \sqrt{h}}\left(H_{W}^{h}-z\right) u \bar{u} d x & =q_{W}^{h}\left(e^{\Phi / \sqrt{h}} u\right) \\
& -h \int_{W} e^{2 \Phi / \sqrt{h}}|\nabla \Phi|^{2}|u|^{2} d x-\operatorname{Re} z \int_{W} e^{2 \Phi / \sqrt{h}}|u|^{2} d x .
\end{aligned}
$$

3.2. Estimates away from the wells. Let $W \subset M$ be a $\Gamma$-invariant open domain (with a regular boundary). We will start with a slight extension of $[7$, Theorem $3.1]$.

Theorem 3.2. There exist constants $C_{0}>0$ and $h_{0}>0$ such that for any $h \in$ $\left(0, h_{0}\right]$ and for any $u \in \operatorname{Dom}\left(q_{W}^{h}\right)$,

$$
h \int_{W}\left[\operatorname{Tr}^{+} B(x)-h^{1 / 4} C_{0}\right]|u(x)|^{2} d x \leq\left(1+h^{1 / 4} C_{0}\right) q_{W}^{h}(u) .
$$

As a consequence of this theorem, we get

$$
\sigma\left(H_{W}^{h}\right) \subset\left[h b_{0}(W)-C h^{5 / 4},+\infty\right), \quad h \in\left(0, h_{0}\right],
$$

with some $C>0$ and $h_{0}>0$.

Proposition 3.3. Let $\Phi \in \mathcal{W}(\bar{W})$. Assume that $K(h)$ is a bounded subset in $\mathbb{C}$ such that $K(h) \subset\left\{z \in \mathbb{C}: \operatorname{Re} z<h\left(b_{0}(W)-\alpha\right)\right\}$ for some $\alpha>0$. If $h>0$ is small enough, then $K(h) \cap \sigma\left(H_{W}^{h}\right)=\emptyset$, and for any $z \in K(h)$ the operator $\left(H_{W}^{h}-z\right)^{-1}$ defines a bounded operator in $L_{\Phi / \sqrt{h}}^{2}(W)$ with

$$
\left\|\left(H_{W}^{h}-z\right)^{-1}\right\|_{\Phi / \sqrt{h}} \leq \frac{C}{h}
$$

uniformly on $z \in K(h)$.

Proof. By Theorem 3.2 and Lemma 3.1, for any $z \in \mathbb{C}$, we have

$$
\begin{aligned}
& \operatorname{Re} \int_{W} e^{2 \Phi / \sqrt{h}}\left(H_{W}^{h}-z\right) u \bar{u} d x \\
& \quad \geq h \int_{W}\left(1+h^{1 / 4} C_{0}\right)^{-1}\left[\operatorname{Tr}^{+} B(x)-h^{1 / 4} C_{0}\right] e^{2 \Phi / \sqrt{h}}|u(x)|^{2} d x \\
& \quad-h \int_{W} e^{2 \Phi / \sqrt{h}}|\nabla \Phi(x)|^{2}|u(x)|^{2} d x-\operatorname{Re} z \int_{W} e^{2 \Phi / \sqrt{h}}|u(x)|^{2} d x
\end{aligned}
$$


that implies

$$
\begin{aligned}
& \operatorname{Re} \int_{W} e^{2 \Phi / \sqrt{h}}\left(H_{W}^{h}-z\right) u \bar{u} d x \\
& \quad \geq h \int_{W} e^{2 \Phi / \sqrt{h}}\left[\operatorname{Tr}^{+} B(x)-|\nabla \Phi(x)|^{2}-\frac{\operatorname{Re} z}{h}\right]|u(x)|^{2} d x+c h^{5 / 4}\|u\|_{\Phi / \sqrt{h}}^{2} \\
& \geq\left(\alpha+c h^{1 / 4}\right) h\|u\|_{\Phi / \sqrt{h}}^{2},
\end{aligned}
$$

and immediately completes the proof.

Corollary 3.4. Under the assumptions of Proposition 3.3, we have

$$
\begin{aligned}
q_{W}\left[e^{\Phi / \sqrt{h}}\left(H_{W}^{h}-z\right)^{-1} v\right]+h\left\|\left(H_{W}^{h}-z\right)^{-1} v\right\|_{\Phi / \sqrt{h}}^{2} & \\
& \leq \frac{C}{h}\|v\|_{\Phi / \sqrt{h}}^{2}, \quad v \in L_{\Phi / \sqrt{h}}^{2}(W) .
\end{aligned}
$$

Proof. By (3.1), for any $h$ small enough one has

$$
\begin{aligned}
q^{h}\left[e^{\Phi / \sqrt{h}}\left(H_{W}^{h}-z\right)^{-1} v\right]= & \operatorname{Re}\left(e^{2 \Phi / \sqrt{h}} v,\left(H_{W}^{h}-z\right)^{-1} v\right) \\
& +h\left\||\nabla \Phi|\left(H_{W}^{h}-z\right)^{-1} v\right\|_{\Phi / \sqrt{h}}^{2} \\
& +\operatorname{Re} z\left\|\left(H_{W}^{h}-z\right)^{-1} v\right\|_{\Phi / \sqrt{h}}^{2} .
\end{aligned}
$$

Now we know that $\operatorname{Re} z<h\left(b_{0}(W)-\alpha\right),|\nabla \Phi|$ is uniformly bounded and

$$
\begin{aligned}
\operatorname{Re}\left(e^{2 \Phi / \sqrt{h}} v,\left(H_{W}^{h}-z\right)^{-1} v\right) & \leq \frac{1}{2}\left(\frac{1}{h}\|v\|_{\Phi / \sqrt{h}}^{2}+h\left\|\left(H_{W}^{h}-z\right)^{-1} v\right\|_{\Phi / \sqrt{h}}^{2}\right) \\
& \leq \frac{C}{h}\|v\|_{\Phi / \sqrt{h}}^{2},
\end{aligned}
$$

that completes the proof.

3.3. Estimates near the wells. In this section, we will assume that $W$ is a relatively compact domain (with smooth boundary) in $\mathcal{F}$ such that

$$
U_{\epsilon_{1}}=\left\{x \in \mathcal{F}: \operatorname{Tr}^{+}(B(x))<b_{0}+\epsilon_{1}\right\}
$$

is contained in $W$ for some $\epsilon_{1}<\epsilon_{0}$.

Proposition 3.5. Assume that $K(h)$ is a bounded subset in $\mathbb{C}$ such that $K(h) \subset$ $\left\{z \in \mathbb{C}: \operatorname{Re} z<h\left(b_{0}+\epsilon_{1}\right)\right\}$ and, if $h>0$ is small enough, then, for any $\epsilon>0$,

$$
\operatorname{dist}\left(K(h), \sigma\left(H_{W}^{h}\right)\right) \geq \frac{1}{C_{\epsilon}} e^{-\epsilon / \sqrt{h}} .
$$

Let $\Phi \in \mathcal{W}(\bar{W})$ such that $\Phi \equiv 0$ on $U_{\epsilon_{1}}$. Then for any $z \in K(h)$ the operator $\left(H_{W}^{h}-z\right)^{-1}$ defines a bounded operator in $L_{\Phi / \sqrt{h}}^{2}(W)$ and for any $\epsilon>0$,

$$
\left\|\left(H_{W}^{h}-z\right)^{-1}\right\|_{\Phi / \sqrt{h}} \leq C_{1, \epsilon} e^{\epsilon / \sqrt{h}}
$$

Proof. For every sufficiently small $\eta>0$, take any $\chi_{1, \eta} \in C_{c}^{\infty}(W)$ such that $\chi_{1, \eta} \equiv 1$ in a neighborhood of $\{x \in W: \Phi(x) \leq 2 \eta\}, \Phi \leq 3 \eta$ on $\operatorname{supp} \chi_{1, \eta}$. Let $\chi_{1, \eta}^{\prime} \in$ $C^{\infty}(W), \chi_{1, \eta}^{\prime} \geq 0$ satisfy $\left(\chi_{1, \eta}\right)^{2}+\left(\chi_{1, \eta}^{\prime}\right)^{2}=1$. We can assume that there exists a constant $C$ such that, for all sufficiently small $\eta>0$,

$$
\eta\left(\left|\nabla \chi_{1, \eta}\right|+\left|\nabla \chi_{1, \eta}^{\prime}\right|\right) \leq C .
$$


Then we have

$$
\begin{aligned}
q_{W}\left(e^{\Phi / \sqrt{h}} u\right)=q\left(\chi_{1, \eta} e^{\Phi / \sqrt{h}} u\right) & +\left(\chi_{1, \eta}^{\prime} e^{\Phi / \sqrt{h}} u\right) \\
& -h^{2}\left\|\left|\nabla \chi_{1, \eta}\right| e^{\Phi / \sqrt{h}} u\right\|^{2}-h^{2}\left\|\left|\nabla \chi_{1, \eta}^{\prime}\right| e^{\Phi / \sqrt{h}} u\right\|^{2} .
\end{aligned}
$$

By (3.1), it follows that

$$
\begin{gathered}
q_{W}\left(\chi_{1, \eta}^{\prime} e^{\Phi / \sqrt{h}} u\right)-h \int_{W} e^{2 \Phi / \sqrt{h}}|\nabla \Phi|^{2}\left|\chi_{1, \eta}^{\prime} u\right|^{2} d x-\operatorname{Re} z \int_{W} e^{2 \Phi / \sqrt{h}}\left|\chi_{1, \eta}^{\prime} u\right|^{2} d x \\
-h^{2}\left\|\left|\nabla \chi_{1, \eta}\right| e^{\Phi / \sqrt{h}} \chi_{1, \eta}^{\prime} u\right\|^{2}-h^{2}\left\|\left|\nabla \chi_{1, \eta}^{\prime}\right| e^{\Phi / \sqrt{h}} \chi_{1, \eta}^{\prime} u\right\|^{2} \\
=\operatorname{Re} \int_{W} e^{2 \Phi / \sqrt{h}}\left(H_{W}^{h}-z\right) u \bar{u} d x-q_{W}\left(\chi_{1, \eta} e^{\Phi / \sqrt{h}} u\right) \\
+h \int_{W} e^{2 \Phi / \sqrt{h}}|\nabla \Phi|^{2}\left|\chi_{1, \eta} u\right|^{2} d x+\operatorname{Re} z \int_{W} e^{2 \Phi / \sqrt{h}}\left|\chi_{1, \eta} u\right|^{2} d x \\
\quad+h^{2}\left\|\left|\nabla \chi_{1, \eta}\right| e^{\Phi / \sqrt{h}} \chi_{1, \eta} u\right\|^{2}+h^{2}\left\|\left|\nabla \chi_{1, \eta}^{\prime}\right| e^{\Phi / \sqrt{h}} \chi_{1, \eta} u\right\|^{2} .
\end{gathered}
$$

Put $\eta=\alpha \sqrt{h}$ with sufficiently large $\alpha>0$. Taking into account the fact that

$$
\left|\nabla \chi_{1, \eta}\right|+\left|\nabla \chi_{1, \eta}^{\prime}\right| \leq C / \eta=C / \alpha \sqrt{h}
$$

we get the following estimate for the right-hand side of (3.2):

$$
\begin{aligned}
h \int_{W} e^{2 \Phi / \sqrt{h}}|\nabla \Phi|^{2}\left|\chi_{1, \eta} u\right|^{2} d x+\operatorname{Re} z \int_{W} e^{2 \Phi / \sqrt{h}}\left|\chi_{1, \eta} u\right|^{2} d x & \\
+h^{2}\left\|\left|\nabla \chi_{1, \eta}\right| e^{\Phi / \sqrt{h}} \chi_{1, \eta} u\right\|^{2}+h^{2}\left\|\left|\nabla \chi_{1, \eta}^{\prime}\right| e^{\Phi / \sqrt{h}} \chi_{1, \eta} u\right\|^{2} & \leq C h\left\|e^{\Phi / \sqrt{h}} \chi_{1, \eta} u\right\|^{2} .
\end{aligned}
$$

From the other side, proceeding as in the proof of Proposition 3.3 and using Theorem 3.2 , we get the estimate for the left-hand side of (3.2):

$$
\begin{gathered}
q_{W}\left(\chi_{1, \eta}^{\prime} e^{\Phi / \sqrt{h}} u\right)-h \int_{W} e^{2 \Phi / \sqrt{h}}|\nabla \Phi|^{2}\left|\chi_{1, \eta}^{\prime} u\right|^{2} d x-\operatorname{Re} z \int_{W} e^{2 \Phi / \sqrt{h}}\left|\chi_{1, \eta}^{\prime} u\right|^{2} d x \\
-h^{2}\left\|\left|\nabla \chi_{1, \eta}\right| e^{\Phi / \sqrt{h}} \chi_{1, \eta}^{\prime} u\right\|^{2}-h^{2}\left\|\left|\nabla \chi_{1, \eta}^{\prime}\right| e^{\Phi / \sqrt{h}} \chi_{1, \eta}^{\prime} u\right\|^{2} \\
\geq h \int_{W} e^{2 \Phi / \sqrt{h}}\left[\operatorname{Tr}^{+} B(x)-|\nabla \Phi(x)|^{2}-\frac{\operatorname{Re} z}{h}-\frac{1}{\alpha^{2}}\right]\left|\chi_{1, \eta}^{\prime} u(x)\right|^{2} d x \\
+c h^{5 / 4}\left\|e^{\Phi / \sqrt{h}} \chi_{1, \eta}^{\prime} u\right\|^{2} \geq C h\left\|e^{\Phi / \sqrt{h}} \chi_{1, \eta}^{\prime} u\right\|^{2} .
\end{gathered}
$$

Thus we get the estimate

$$
c h\left\|e^{\Phi / \sqrt{h}} u\right\|^{2} \leq \operatorname{Re} \int_{W} e^{2 \Phi / \sqrt{h}}\left(H_{W}^{h}-z\right) u \bar{u} d x+C h\left\|e^{\Phi / \sqrt{h}} \chi_{1, \eta} u\right\|^{2} .
$$

It remains to show that, for any $\epsilon>0$,

$$
\left\|e^{\Phi / \sqrt{h}} \chi_{1, \eta} u\right\| \leq C_{\epsilon} e^{\epsilon / \sqrt{h}}\left\|e^{\Phi / \sqrt{h}}\left(H_{W}^{h}-z\right) u\right\|,
$$

or equivalently,

$$
\left\|\chi_{1, \eta}\left(H_{W}^{h}-z\right)^{-1} u\right\|_{\Phi / \sqrt{h}} \leq C_{\epsilon} e^{\epsilon / \sqrt{h}}\|u\|_{\Phi / \sqrt{h}}, \quad u \in L_{\Phi / \sqrt{h}}^{2}(W) .
$$


For this, we choose a function $\chi_{2, \eta} \in C_{c}^{\infty}(W)$ such that $\chi_{2, \eta} \equiv 1$ in a neighborhood of $\{x \in W: \Phi(x) \leq \eta\}, \Phi \leq 2 \eta$ on supp $\chi_{2, \eta}$. In particular, $\chi_{1, \eta} \equiv 1$ on $\operatorname{supp} \chi_{2, \eta}$. We can assume that there exists a constant $C$ such that for all sufficiently small $\eta>0$

$$
\eta\left|\nabla \chi_{2, \eta}\right|+\eta^{2}\left|\Delta \chi_{2, \eta}\right| \leq C
$$

Let $M_{0}=\{x \in W: \Phi(x) \geq 2 \eta\}$. Then we have

$$
\begin{aligned}
\left(H_{W}^{h}-z\right)^{-1} u=\left(1-\chi_{2, \eta}\right) & \left(H_{M_{0}}^{h}-z\right)^{-1}\left(1-\chi_{1, \eta}\right) u+\left(H_{W}^{h}-z\right)^{-1} \chi_{1, \eta} u \\
+ & \left(H_{W}^{h}-z\right)^{-1} \chi_{1, \eta}\left[H_{W}^{h}, \chi_{2, \eta}\right]\left(H_{M_{0}}^{h}-z\right)^{-1}\left(1-\chi_{1, \eta}\right) u .
\end{aligned}
$$

We consider three terms in the right hand side of the last identity separately. For the first one we use Proposition 3.3 and obtain

$$
\left\|\chi_{1, \eta}\left(1-\chi_{2, \eta}\right)\left(H_{M_{0}}^{h}-z\right)^{-1}\left(1-\chi_{1, \eta}\right) u\right\|_{\Phi / \sqrt{h}} \leq \frac{C}{h}\|u\|_{\Phi / \sqrt{h}} .
$$

For the second term, since $\Phi \leq 3 \eta$ on supp $\chi_{1, \eta}$, we have

$$
\left\|\chi_{1, \eta}\left(H_{W}^{h}-z\right)^{-1} \chi_{1, \eta} u\right\|_{\Phi / \sqrt{h}} \leq e^{3 \alpha}\left\|\left(H_{W}^{h}-z\right)^{-1} \chi_{1, \eta} u\right\| .
$$

By the assumptions and the fact that $\Phi \geq 0$, it follows that

$$
\left\|\left(H_{W}^{h}-z\right)^{-1} \chi_{1, \eta} u\right\| \leq e^{\epsilon / \sqrt{h}}\left\|\chi_{1, \eta} u\right\| \leq C_{1} e^{\epsilon / \sqrt{h}}\|u\|_{\Phi / \sqrt{h}} .
$$

So we get for the second term

$$
\left\|\chi_{1, \eta}\left(H_{W}^{h}-z\right)^{-1} \chi_{1, \eta} u\right\|_{\Phi / \sqrt{h}} \leq C_{2} e^{\epsilon / \sqrt{h}}\|u\|_{\Phi / \sqrt{h}} .
$$

For the third term we put $w=\left(H_{M_{0}}^{h}-z\right)^{-1}\left(1-\chi_{1, \eta}\right) u$. By (3.6), it follows that

$$
\left\|\chi_{1, \eta}\left(H_{W}^{h}-z\right)^{-1} \chi_{1, \eta}\left[H_{W}^{h}, \chi_{2, \eta}\right] w\right\|_{\Phi / \sqrt{h}} \leq C_{1} e^{\epsilon / \sqrt{h}}\left\|\left[H_{W}^{h}, \chi_{2, \eta}\right] w\right\|_{\Phi / \sqrt{h}} .
$$

Now we have

$$
\left[H_{W}^{h}, \chi_{2, \eta}\right] w=2 i h d \chi_{2, \eta} \cdot(i h d+\mathbf{A}) w+h^{2} \Delta \chi_{2, \eta} w .
$$

Therefore, taking into account (3.4), we get

$$
\begin{aligned}
\left\|\left[H_{W}^{h}, \chi_{2, \eta}\right] w\right\|_{\Phi / \sqrt{h}}^{2} & \leq C\left(h\|(i h d+\mathbf{A}) w\|_{\Phi / \sqrt{h}}^{2}+h^{2}\|w\|_{\Phi / \sqrt{h}}^{2}\right) \\
& \leq C\left(h q_{W}\left[e^{\Phi / \sqrt{h}} w\right]+h^{2}\|w\|_{\Phi / \sqrt{h}}^{2}\right) .
\end{aligned}
$$

By Corollary 3.4, we have

$$
\begin{aligned}
\left\|\left[H_{W}^{h}, \chi_{2, \eta}\right] w\right\|_{\Phi / \sqrt{h} \leq}^{2} \leq & C\left(h q_{W}\left[e^{\Phi / \sqrt{h}}\left(H_{M_{0}}^{h}-z\right)^{-1}\left(1-\chi_{1, \eta}\right) u\right]\right. \\
& \left.+h^{2}\left\|\left(H_{M_{0}}^{h}-z\right)^{-1}\left(1-\chi_{1, \eta}\right) u\right\|_{\Phi / \sqrt{h}}^{2}\right) \\
\leq & C\left\|\left(1-\chi_{1, \eta}\right) u\right\|_{\Phi / \sqrt{h}}^{2} \leq C\|u\|_{\Phi / \sqrt{h}}^{2} .
\end{aligned}
$$


So we get for the third term

$$
\begin{aligned}
&\left\|\chi_{1, \eta}\left(H_{W}^{h}-z\right)^{-1} \chi_{1, \eta}\left[H_{W}^{h}, \chi_{2, \eta}\right]\left(H_{M_{0}}^{h}-z\right)^{-1}\left(1-\chi_{1, \eta}\right) u\right\|_{\Phi / \sqrt{h}} \\
& \leq C_{3, \epsilon} e^{\epsilon / \sqrt{h}}\|u\|_{\Phi / \sqrt{h}} .
\end{aligned}
$$

Now (3.3) follows by adding the estimates (3.5), (3.6) and (3.7).

Corollary 3.6. Under the assumptions of Proposition 3.5, we have, for any $\epsilon>0$,

$$
\begin{aligned}
q_{W}\left[e^{\Phi / \sqrt{h}}\left(H_{W}^{h}-z\right)^{-1} v\right]+h\left\|\left(H_{W}^{h}-z\right)^{-1} v\right\|_{\Phi / \sqrt{h}}^{2} & \\
& \leq C_{2, \epsilon} e^{\epsilon / \sqrt{h}}\|v\|_{\Phi / \sqrt{h}}^{2}, \quad v \in L_{\Phi / \sqrt{h}}^{2}(W) .
\end{aligned}
$$

3.4. Proof of Theorem 2.1. Let us assume that (1.1) and (1.2) are satisfied. We have

$$
\left\{x \in \mathcal{F}: \operatorname{Tr}^{+}(B(x))<b_{0}+\epsilon_{2}\right\}=U_{\epsilon_{1}}=\bigcup_{j=1}^{N} U_{j, \epsilon_{2}},
$$

where $U_{j, \epsilon_{2}} \subset \mathcal{F}, j=1,2, \cdots, N$, are relatively compact, connected and pairwise disjoint domains such that $U_{j, \epsilon_{2}} \cap \partial \mathcal{F}=\emptyset$. Let $M_{j}=\overline{U_{j, \epsilon_{2}}}, j=1,2, \cdots, N$. Theorem 2.1 follows immediately from the following.

Proposition 3.7. Assume that $K(h)$ is a bounded subset in $\mathbb{C}$ such that $K(h) \subset$ $\left\{z \in \mathbb{C}: \operatorname{Re} z<h\left(b_{0}+\epsilon_{1}\right)\right\}$ and, if $h>0$ is small enough, then, for any $\epsilon>0$,

$$
\operatorname{dist}\left(K(h), \sigma\left(H_{M_{j}}^{h}\right)\right) \geq \frac{1}{C_{\epsilon}} e^{-\epsilon / \sqrt{h}}, \quad j=1,2, \cdots, N .
$$

Then, for any $h>0$ small enough, $K(h) \cap \sigma\left(H^{h}\right)=\emptyset$.

Proof. Take any $\eta>0$ such that $\epsilon_{1}+3 \eta<\epsilon_{2}$. Let

$$
M_{0}=M \backslash \bigcup_{\gamma \in \Gamma} \bigcup_{j=1}^{N} \gamma\left(U_{j, \epsilon_{1}+\eta}\right)=\left\{x \in M: \operatorname{Tr}^{+}(B(x)) \geq b_{0}+\epsilon_{1}+\eta\right\} .
$$

Take any function $\phi_{j} \in C_{c}^{\infty}(M)$ such that $\operatorname{supp} \phi_{j} \subset U_{j, \epsilon_{1}+2 \eta}, \phi_{j} \equiv 1$ on $U_{j, \epsilon_{1}+\eta}$. Let

$$
\phi_{0}=1-\sum_{\gamma \in \Gamma} \sum_{j=1}^{N} \gamma^{*} \phi_{j}
$$

Then $\operatorname{supp} \phi_{0} \subset M_{0}$. Let $\psi_{j} \in C_{c}^{\infty}(M), j=1,2, \cdots, N$, such that $\operatorname{supp} \psi_{j} \subset$ $U_{j, \epsilon_{1}+3 \eta}$, and $\psi_{j} \equiv 1$ in a neighborhood of $U_{j, \epsilon_{1}+2 \eta}$. Take any $\Gamma$-periodic function $\psi_{0} \in C^{\infty}(M)$ such that $\operatorname{supp} \psi_{0} \subset M_{0}$, and $\psi_{0} \equiv 1$ in a neighborhood of $M \backslash \bigcup_{\gamma \in \Gamma} \bigcup_{j=1}^{N} \gamma\left(U_{j, \epsilon_{1}+2 \eta}\right)$. In particular, we have $\phi_{j} \psi_{j}=\phi_{j}$, for $j=0,1,2, \cdots, N$.

Recall that the magnetic translations $T_{\gamma}, \gamma \in \Gamma$, are unitary operators in $L^{2}(M)$, which commute with the periodic magnetic Schrödinger operator $H^{h}$ :

$$
T_{\gamma} H^{h}=H^{h} T_{\gamma}, \quad \gamma \in \Gamma,
$$

and each $T_{\gamma}$ takes $L^{2}(\mathcal{F})$ to $L^{2}(\gamma \mathcal{F})$ (see for instance $[17,15]$ and the references therein for more details). They satisfy

$$
T_{e}=\mathrm{id}, \quad T_{\gamma_{1}} T_{\gamma_{2}}=\sigma\left(\gamma_{1}, \gamma_{2}\right) T_{\gamma_{1} \gamma_{2}}, \quad \gamma_{1}, \gamma_{2} \in \Gamma .
$$


Here $\sigma$ is a 2-cocycle on $\Gamma$, i.e. $\sigma: \Gamma \times \Gamma \rightarrow U(1)$ such that

$$
\begin{aligned}
\sigma(\gamma, e) & =\sigma(e, \gamma)=1, \quad \gamma \in \Gamma ; \\
\sigma\left(\gamma_{1}, \gamma_{2}\right) \sigma\left(\gamma_{1} \gamma_{2}, \gamma_{3}\right) & =\sigma\left(\gamma_{1}, \gamma_{2} \gamma_{3}\right) \sigma\left(\gamma_{2}, \gamma_{3}\right), \quad \gamma_{1}, \gamma_{2}, \gamma_{3} \in \Gamma .
\end{aligned}
$$

For any $h>0$ small enough and any $z \in K(h)$, define a bounded operator $R^{h}(z)$ in $L^{2}(M)$ as

$$
R^{h}(z)=\sum_{j=1}^{N} \sum_{\gamma \in \Gamma} T_{\gamma} \psi_{j}\left(H_{M_{j}}^{h}-z\right)^{-1} \phi_{j} T_{\gamma}^{*}+\psi_{0}\left(H_{M_{0}}^{h}-z\right)^{-1} \phi_{0} .
$$

Then

$$
\left(H^{h}-z\right) R^{h}(z)=I-K^{h}(z),
$$

where

$$
K^{h}(z)=\sum_{j=1}^{N} \sum_{\gamma \in \Gamma} T_{\gamma}\left[H^{h}, \psi_{j}\right]\left(H_{M_{j}}^{h}-z\right)^{-1} \phi_{j} T_{\gamma}^{*}+\left[H^{h}, \psi_{0}\right]\left(H_{M_{0}}^{h}-z\right)^{-1} \phi_{0} .
$$

Lemma 3.8. There exist $C, c>0$ such that, for any $h>0$ small enough and $z \in K(h)$, the operator $K^{h}(z)$ defines a bounded operator in $L^{2}(M)$ with the norm estimate

$$
\left\|K^{h}(z)\right\| \leq C e^{-c / \sqrt{h}} .
$$

Proof. For any $j=1,2, \cdots, N$, consider a weight function $\Phi_{j} \in \mathcal{W}\left(M_{j}\right)$ given by $\Phi_{j}(x)=d_{U_{j, \epsilon_{1}+2 \eta}}\left(x, U_{j, \epsilon_{1}+2 \eta}\right)$. By construction, $\Phi_{j}(x) \geq c_{j}>0$ on $\operatorname{supp} d \psi_{j}$, $\Phi_{j}(x) \equiv 0$ on $\operatorname{supp} \phi_{j}$. For any $w \in \operatorname{Dom} H^{h}$, we have

$$
\left[H^{h}, \psi_{j}\right] w=2 i h d \psi_{j} \cdot(i h d+\mathbf{A}) w+h^{2} \Delta \psi_{j} w .
$$

This implies the estimate

$$
\begin{aligned}
\left\|\left[H^{h}, \psi_{j}\right] w\right\|_{\Phi_{j} / \sqrt{h}}^{2} & \leq C\left(h\|(i h d+\mathbf{A}) w\|_{\Phi_{j} / \sqrt{h}}^{2}+h^{2}\|w\|_{\Phi_{j} / \sqrt{h}}^{2}\right) \\
& \leq C\left(h q_{M_{j}}\left[e^{\Phi_{j} / \sqrt{h}} w\right]+h^{2}\|w\|_{\Phi_{j} / \sqrt{h}}^{2}\right) .
\end{aligned}
$$

Therefore, for any $u \in L^{2}(M)$, we obtain

$$
\begin{aligned}
\left\|\left[H^{h}, \psi_{j}\right]\left(H_{M_{j}}^{h}-z\right)^{-1} \phi_{j} u\right\|_{L^{2}(M)}^{2}= & \left\|\left[H^{h}, \psi_{j}\right]\left(H_{M_{j}}^{h}-z\right)^{-1} \phi_{j} u\right\|_{L^{2}\left(M_{j}\right)}^{2} \\
\leq & e^{-c_{j} / \sqrt{h}}\left\|\left[H^{h}, \psi_{j}\right]\left(H_{M_{j}}^{h}-z\right)^{-1} \phi_{j} u\right\|_{\Phi_{j} / \sqrt{h}}^{2} \\
\leq & C e^{-c_{j} / \sqrt{h}}\left(h q_{M_{j}}\left[e^{\Phi_{j} / \sqrt{h}}\left(H_{M_{j}}^{h}-z\right)^{-1} \phi_{j} u\right]\right. \\
& \left.+h^{2}\left\|\left(H_{M_{j}}^{h}-z\right)^{-1} \phi_{j} u\right\|_{\Phi_{j} / \sqrt{h}}^{2}\right) .
\end{aligned}
$$

It follows from Corollary 3.6 that, for any $\epsilon>0$,

$$
\begin{aligned}
\left\|\left[H^{h}, \psi_{j}\right]\left(H_{M_{j}}^{h}-z\right)^{-1} \phi_{j} u\right\|_{L^{2}(M)} & \leq C_{\epsilon} e^{-\left(c_{j}-\epsilon\right) / \sqrt{h}}\left\|\phi_{j} u\right\|_{\Phi_{j} / \sqrt{h}} \\
& =C_{\epsilon} e^{-\left(c_{j}-\epsilon\right) / \sqrt{h}}\left\|\phi_{j} u\right\|_{L^{2}\left(M_{j}\right)} \\
& =C_{\epsilon} e^{-\left(c_{j}-\epsilon\right) / \sqrt{h}}\|u\|_{L^{2}(M)} .
\end{aligned}
$$

Similarly, using Corollary 3.4, one can get

$$
\left\|\left[H^{h}, \psi_{0}\right]\left(H_{M_{0}}^{h}-z\right)^{-1} \phi_{0} u\right\|_{L^{2}(M)} \leq C_{0} e^{-c_{0} / \sqrt{h}}\|u\|_{L^{2}(M)} .
$$


Taking into account that the sets $\gamma\left(\operatorname{supp} \phi_{j}\right)$ with $j=1,2, \cdots, N$ and $\gamma \in \Gamma$ are disjoint, we get

$$
\begin{aligned}
\left\|K^{h}(z) u\right\| & \leq C e^{-c / \sqrt{h}}\left(\sum_{j=1}^{N} \sum_{\gamma \in \Gamma}\left\|\phi_{j} T_{\gamma}^{*} u\right\|+\left\|\phi_{0} u\right\|\right) \\
& \leq C_{1} e^{-c / \sqrt{h}}\|u\| .
\end{aligned}
$$

This completes the proof.

It follows from Lemma 3.8 that, for all sufficiently small $h>0$ and $z \in K(h)$, the operator $I+K^{h}(z)$ is invertible in $L^{2}(M)$. Then the operator $H^{h}-z$ is invertible in $L^{2}(M)$ with

$$
\left(H^{h}-z\right)^{-1}=R^{h}(z)\left(I-K^{h}(z)\right)^{-1},
$$

and $K(h) \cap \sigma\left(H^{h}\right)=\emptyset$ as desired.

\section{REFERENCES}

[1] S. Agmon, Lectures on exponential decay of solutions of second-order elliptic equations. Mathematical Notes 29, Princeton University Press, Princeton, 1982. MR745286 (85f:35019)

[2] J. Brüning, S. Yu. Dobrokhotov, K. V. Pankrashkin. The spectral asymptotics of the twodimensional Schrödinger operator with a strong magnetic field. I. Russ. J. Math. Phys. 9 (2002), no. 1, 14-49; II. Russ. J. Math. Phys. 9 (2002), no. 4, 400-416 (see also e-print version math-ph/0411012). MR1965505 (2005e:81052)

[3] U. Carlsson, An infinite number of wells in the semi-classical limit. Asymptotic Anal. 3 (1990), no. 3, 189-214. MR1076447 (91i:35134)

[4] H. D. Cornean, G. Nenciu, Two dimensional magnetic Schrödinger operators: width of mini bands in the tight binding approximation, Ann. Henri Poincaré 1 (2000), 203-222. MR1770797 (2001g:81059)

[5] M. Dimassi, J. Sjöstrand, Spectral asymptotics in the semi-classical limit. London Mathematical Society Lecture Notes Series, 268, Cambridge University Press, Cambridge, 1999. MR1735654 (2001b:35237)

[6] R. L. Frank, On the tunneling effect for magnetic Schrödinger operators in antidot lattices. Asymptot. Anal. 48 (2006), no. 1-2, 91-120. MR2233380 (2007b:81074)

[7] B. Helffer, A. Mohamed, Semiclassical analysis for the ground state energy of a Schrödinger operator with magnetic wells. J. Funct. Anal. 138 (1996), 40-81. MR1391630 (97h:35177)

[8] B. Helffer, J. Sjöstrand, Multiple wells in the semiclassical limit. I. Comm. Partial Differential Equations 9 (1984), 337-408. MR740094 (86c:35113)

[9] B. Helffer, J. Sjöstrand, Puits multiples en limite semi-classique. II. Interaction moléculaire. Symétries. Perturbation. Ann. Inst. H. Poincaré Phys. Théor. 42, no. 2 (1985), 127-212. MR798695 (87a:35142)

[10] B. Helffer, J. Sjöstrand, Effet tunnel pour l'équation de Schrödinger avec champ magnétique. Ann. Scuola Norm. Sup. Pisa Cl. Sci. (4) 14 (1987), 625-657. MR963493 (91c:35043)

[11] B. Helffer, J. Sjöstrand, Analyse semi-classique pour l'équation de Harper (avec application à l'équation de Schrödinger avec champ magnétique). Mém. Soc. Math. France (N.S.) 34 (1988). MR1003937 (91d:81024)

[12] B. Helffer, J. Sjöstrand, Équation de Schrödinger avec champ magnétique et équation de Harper, In: Schrödinger operators (Sønderborg, 1988), Lecture Notes in Phys., 345, Springer, Berlin, 1989, pp. 118-197. MR1037319 (91g:35078)

[13] R. Hempel, I. Herbst, Strong magnetic fields, Dirichlet boundaries, and spectral gaps. Commun. Math. Phys. 169 (1995), 237-259. MR1329195 (96a:81026)

[14] I. Herbst, S. Nakamura. Schrödinger operators with strong magnetic fields: quasi-periodicity of spectral orbits and topology. In: Differential operators and spectral theory, Amer. Math. Soc. Transl. Ser. 2, 189, Amer. Math. Soc., Providence, RI, 1999, pp. 105-123. MR1730507 (2001g:81066) 
[15] Yu. A. Kordyukov, V. Mathai, M. Shubin, Equivalence of projections in semiclassical limit and a vanishing theorem for higher traces in K-theory. J. Reine Angew. Math. 581 (2005), 193-236. MR2132676 (2007b:58040)

[16] Yu. A. Kordyukov, Spectral gaps for periodic Schrödinger operators with strong magnetic fields. Commun. Math. Phys. 253 (2005), 371-384. MR2140253 (2006h:81079)

[17] V. Mathai, M. Shubin, Semiclassical asymptotics and gaps in the spectra of magnetic Schrödinger operators. Geometriae Dedicata 91 (2002), 155-173. MR1919898 (2004f:58040)

[18] S. Nakamura, Band spectrum for Schrödinger operators with strong periodic magnetic fields. In: Partial differential operators and mathematical physics (Holzhau, 1994), Operator Theory: Advances and Applications. vol. 78, Birkhäuser, Basel, 1995, pp. 261-270. MR1365340 (97a:81038)

Département de Mathématiques, Bâtiment 425, F91405 Orsay Cédex, France

E-mail address: Bernard.Helffer@math.u-psud.fr

Institute of Mathematics, Russian Academy of Sciences, 112 Chernyshevsky str., 450077 UfA, Russia

E-mail address: yurikor@matem.anrb.ru 